\title{
OVEREXPRESSION OF OSHox-6 GENE ENHANCED TILLER NUMBER IN RICE BUT INDUCED YIELD PENALTY
}

\author{
Syamsidah Rahmawati*, Chairunnisa, Eva Erdayani, Satya Nugroho and Amy Estiati \\ Research Center for Biotechnology, Indonesian Institute of Sciences (LIPI)
}

\begin{abstract}
OsHox-6, belongs to the transcription factor homeodomain leucine zipper (HD-Zip) protein sub-family I, has unknown function. This study was aimed to characterize the phenotypes of two homozygous transgenic rice lines (S29-62-2 and S.40.4-158-1) containing an extra copy of OsHox-6 gene under the control of a rice constitutive promoter, OsLEA3, and to evaluate their tolerance to water stress. A real-time quantitative PCR (qRT-PCR) showed that the transcript expression of OsHox-6 gene in the transgenic lines increased 5-10 folds under a normal irrigation and 10-20 folds after exposure to water stress conditions as compared to its wild type control. Transgenic plants overexpressing OsHox-6 exhibited phenotypic alteration at the normal irrigation by inducing tiller formation, suggesting a decrease in the apical dominance. Transgenic plants also showed significant enhancement in the total grain number, however, the number of empty grains also increased significantly $(\sim 16-$ $22 \%$ ). After imposed to the water stress, the number of empty grains in the transgenic lines was even higher (up to $83 \%$ in average). Furthermore, observations on the water loss rates, relative water contents and drought resistance indices (DRI) suggested that the overexpression of $O s H o x-6$ did not significantly increase tolerance to water stress. Further research is required to reveal the detailed mechanisms of OsHox-6 in response to water and other abiotic stresses.
\end{abstract}

Keywords: rice, drought, IR64 Sub1, HD-Zip, OsHox-6

*Corresponding author: Cibinong Science Center, Jl. Raya Bogor Km. 46, Cibinong 16911, Indonesia Tel. +62-21-8754587, Fax. +62-21-87754588

e-mail: syamsidah_rahmawati@yahoo.com

\section{Introduction}

Homeodomain leucine zipper (HD-Zip) is one of the transcription factors found uniquely in plants, containing an essential homeodomain region for DNA binding and a leucin zipper motif that helps protein dimerization. The protein dimerization is required to allow the DNA binding processes to take place. This HD-Zip transcription factors are a large family which are divided into IV sub-families based on the homeodomain DNA binding specificity, the structure and function of genes, and the presence of other motives (Ariel et al., 2007).

Previous studies on different plant species showed that the HD-Zip genes have highly diverse functions. Some of the well-known HD-Zip proteins are GLABRA, PHABULOSA, and REVOLUTA. GLABRA belong to the HD-zip sub-famili IV, while PHABULOSA, CORONA and REVOLUTA are members of HD-Zip sub-families III. The GLABRA genes are important in the formation of trichomes (Rerie et al., 1994), whereas the REVOLUTA, PHABULOSA and CORONA genes play important roles in the development of leaves and vascular tissues, and in the initiation of meristems (Prigge et al., 2005).

The HD-Zip genes, particularly sub-families I and II, have been of particular interest of researchers, especially for their role in the mechanism of drought tolerance in plants. Five HD-Zip genes $(\mathrm{CpHB}-3,-4,-5,-6,-7)$ of Craterostigma plantagineum, a desert and extremely drought tolerant plant, were found to be regulated during dehydration that lead to a desiccation tolerance (Deng et al., 2002). Previously, Frank et al. (1998) isolated 2 HDZip genes $(C p H B-1 \&-2)$ of $C$. plantagineum which were responsive to dehydration at the early stages of the treatment, however, no further reports on the functions or any tolerance mechanisms related to these HD-Zip genes in response to water stress.

A promising report came from Dezar et al. (2005) who studied the Hahb-4 gene (HD-Zip 
sub-families I) of the sunflower (Helianthus annuus). Overexpression of Hahb-4 driven by CaMV35S promoter in Arabidopsis thaliana increased its tolerance to water stress as indicated by higher survival rates compared to the untransformed parental lines. This report suggested the potential use of HD-Zip genes in developing drought-tolerant plants.

In 2007, Ariel et al. has isolated 26 HD-Zip genes belonging to the sub-families I and II from the model plant Arabidopsis thaliana. Four of them, AtHB-5, -6, -7, and -12, were regulated during dehydration. Water stress down regulated the expression of AtHB-5 and 6 genes, but up regulated the expression of AtHB-7 and -12. Previously, Hjellstrom et al. (2003) and Olsson et al. (2004) reported that $A t H B-7$ and -12 play an important role in cell elongation. Overexpression of $A t H B-7$ and -12 lead the plants to become shorter due to reduction in their cell length.

Information on the function and regulation mechanisms of HD-Zip genes in rice is still limited. HD-Zip transcription factors in rice plant were just actively studied in recent ten years. An earlier study came from Agalou et al. (2008) who found as many as 31 of HD-Zip genes in rice that belong to the sub-families I, II, and III. Agalou et al. reported that 8 out of 31 rice HD-zip genes studied, i.e OsHox-4, -6, $-11,-19,-20,-22,-24$, and -27 that belong to the sub-family I and II, were regulated during dehydration. OsHox-4 genes played a role in the elongation and enlargement of the vessel cells (Agalou et al. 2008). Overexpression of OsHox-4 caused a semi-dwarf phenotype and significant reduction in the internode length and the tiller number (Agalou et al. 2008; Dai et al. 2008). OsHox-22 was predicted to play an important role in the ABA biosynthesis. Overexpression of OsHox-22 increased sensitivity to $\mathrm{ABA}$ and dehydration. Down regulation of rice OsHox-22, however, significantly increased rice drought tolerance at the seedling stage (Zhang et al. 2012). Similar results also came from Bhattacharjee et al. (2016) who worked on OsHox-22 and -24. Overexpression of OsHox-22 and -24 in A. thaliana caused greater plant sensitivity to ABA, dehydration and salt stresses. Although HD-Zip transcription factors have been studied in the past two decades, their functions and mechanisms of action in plants, especially in response to water stress, remain unclear. Further studies on the other members of rice
HD-zip genes are also required to gain more comprehensive understanding on the roles of HD-zip genes in drought stress. The findings will be very important to reveal the potential of these genes as candidates for the development of drought tolerant crops.

In the present work, the function of OsHox6 gene in response to water stress was studied. A previous study showed that the transcript levels of OsHox-6 gene were increased during water stress conditions, accumulated in rice nodes, roots and pedicels (Agalou et al. 2008; Rahmawati, 2012). Previously, we have transformed rice $\mathrm{cv}$ IR64 Sub1 with pCAMBIA1301H carrying the OsHox-6 gene under the control of OsLEA3 as a droughtinducible promoter and resulted several homozygous transgenic lines. OsLEA3 was known to perform high activity in water stress conditions (Xiao et al., 2007). We then selected two homozygous lines overexpressing OsHox-6 for further analyses. The phenotypes of the transgenic lines overexpressing OsHox-6 and their tolerance to water stress were evaluated.

\section{Materials and Methods}

\section{Plant Materials}

Two homozygous transgenic lines (S29-622, and S.40.4-158-1) of rice (Oryza sativa L. ssp indica cv IR64 Sub1) carrying the OsHox-6 gene under the control of OsLEA3 promoter were used. The untransformed rice IR64 Sub1 was used as a control in all experiments.

\section{qRT-PCR}

Total RNA was isolated from the young leaves of transgenic and non-transgenic plants before and after air-dried for 1 hour at a room temperature in the lab. Total RNA was extracted from leaves using Trizol LS® Reagent (Invitrogen), following the manufacturer's protocol. Before extraction, total RNA samples were treated with RNasefree DNase I (Thermo Scientific) to remove DNA contaminants. For the quantitative Real Time PCR, KAPA SYBR FAST ${ }^{\circledR}$ one-step qRT-PCR kit (Invitrogen) was used. The qRTPCR reaction mixture contained $5 \mu$ KAPA SYBR FAST buffer, $0.2 \mu \mathrm{l}$ each $10 \mu \mathrm{M}$ forward and reverse primers, $0.2 \mu \mathrm{l} 10 \mu \mathrm{M}$ dUTP, $0.2 \mu$ KAPA RT-mix, and 40 ng RNA 
sample, in a total volume of $10 \mu \mathrm{l}$. Two pairs of primers (Table 1) were used to amplify OsHox6 and actin transcripts, respectively, according to Agalou et al. (2008). Each sample was prepared in duplicates. Template RNA was initially reverse transcribed into cDNA at $42^{\circ} \mathrm{C}$ for 5 minutes. The cDNA was then denatured at $95^{\circ} \mathrm{C}$ for 5 minutes, followed by 40 cycles of PCR amplification in the following conditions : a 3-s denaturation at $95^{\circ} \mathrm{C}$, a 30 -s annealing at $60^{\circ} \mathrm{C}$, and a 30 -s primer extension at $72^{\circ} \mathrm{C}$ to allow completion of primer extension. Quantitative RT-PCR results were analyzed using the software provided by the $\mathrm{Eco}^{\mathrm{TM}}$ Illumina instrument.

Table 1. The sequences of primers used in the qRT-PCR analysis of OsHox-6 expression

\begin{tabular}{lcc}
\hline No. & Primer & Sequence 5' $\rightarrow$ 3 \\
\hline 1. & OsActin fwd & $\begin{array}{c}\text { CTG GGT TCG CCG } \\
\text { GAG ATG AT }\end{array}$ \\
2. & OsActin rev & $\begin{array}{c}\text { TGA GAT CAC GCC } \\
\text { CAG CAA GG }\end{array}$ \\
3. & Oshox6rtF & $\begin{array}{c}\text { GGC CGT CGT CCA } \\
\text { CGG A }\end{array}$ \\
4. & Oshox6rtR & $\begin{array}{c}\text { TCG CTC TCG AAT } \\
\text { TCC CAC C }\end{array}$ \\
\hline
\end{tabular}

\section{Morphological Characterization of} Transgenic Lines

Wild type (WT) and transgenic rice lines were grown in pots, one plant per pot in five biological replicates. All plants were grown in standard irrigation conditions. Fertilizers were applied as recommended. Pest and disease control were carried out when necessary. Observation on agronomic characteristics, i.e. plant height, tiller number, number of productive tillers, flowering time (the time when the number of flowering plants are more than $50 \%$ ), total grain number, filled grain number, and weight of 1000 seeds were performed for the mature plants at the reproductive stage.

\section{Quantification of Water-loss at the Seedling Stage}

Quantification of water loss was carried out according to $\mathrm{Yu}$ et al. (2008). The shoots of 21-day old WT and transgenic seedlings were detached and weighed immediately $\left(\mathrm{FW}_{0}\right)$. The leaves were then incubated in Petri dishes at a room temperature. The fresh weight $(\mathrm{FW})$ was measured at $10 \mathrm{~min}$ interval for two hours. The rates of water loss were calculated from the equation:

Water loss $(\%)=\left(\mathrm{FW}_{0}-\mathrm{FW}\right) /\left(\mathrm{FW}_{0}\right) \times 100 \%$.

\section{Drought Resistance Test at the Generative Stage}

Plants were grown in PVC pots $(\phi 20 \mathrm{~cm}$ and height $80 \mathrm{~cm}$ ) during a dry season (AprilAugust 2012) in a green house. Each pot was filled with $16 \mathrm{~kg}$ of soil, sand and cocopeat (2:1:1). Three seedlings were transplanted into each pot and after three weeks only one seedling which grew best was left per pot. Plants were fertilized with $0.4 \mathrm{~g}$ TSP and $0.3 \mathrm{~g}$ $\mathrm{KCl}$ a day after transplanting while $0.3 \mathrm{~g} \mathrm{~N}$ was added at the week 1, 4, and 6 after transplanting. All the plants were grown normally until the time of imposing the water treatment ( 9 weeks after transplanting). The plants were then divided into two sets of treatments with three replicates of each line. The dry down experiment was done according to Kholova et al. (2010). A day before the water-stress treatment, the soil was saturated with water and allowed to drain overnight (day $0)$. In the morning of the following day, the upper pots were covered with transparent plastic bags that wrap around the pots and the stems. The pots were weighed in every morning during the first three days, and after that, the pots were weighed every two days. One set of the treatment was maintained under a well-watered (WW) condition by daily rewatering until the initial weight was reached. Another set was gradually exposed to water stress (WS) by partially compensating water loss from transpiration (plants were allowed to lose no more than $200 \mathrm{~g}$ each day). The experiment was terminated when the transpiration of WS plants $<10 \%$ of WW plants. The duration of the water stress experiment was 43 days. Plants were then rehydrated, and at harvest time, agronomic characteristic (i.e. plant height, tiller number, number of productive tillers), biomass, and yield were recorded. After the harvest, the fraction of transpirable soil water (FTSW) for each day was calculated using the equation:

FTSW $=$ (pot weight of day $n$-final pot weight)/ (initial pot weight-final pot weight).

The tolerance levels of transgenic plants were evaluated by looking at the resistance index (Drought Resistance Index, DRI) using the equation: DRI $=(\mathrm{Ys} / \mathrm{Yn}) /(\mathrm{Ms} / \mathrm{Mn})$. Ys and $\mathrm{Yn}$ are plant yield or biomass under stress 
(WS) and normal (WW) condition respectively, while Ms and Mn are the average yield or biomass across the genotypes tested consecutively under stress and normal conditions (Fischer \& Maurer, 1978).

\section{Quantification of Relative Water Content (RWC)}

RWC of the leaves was measured at day-0 and day-28 after exposure to water stress treatments. Fully expanded leaves were cut from plants and their fresh weight $(\mathrm{FW})$ were recorded immediately. The leaves were then immersed in distilled water for $16 \mathrm{~h}$ and the turgid weight (TW) values were recorded. The leaves were further dried in an oven for $72 \mathrm{~h}$ at $80^{\circ} \mathrm{C}$ and the dry weight (DW) values were recorded. The RWC values were calculated by using the equation: RWC $(\%)=(\mathrm{FW}-$ DW)/(TW-DW) x $100 \%$.

\section{Statistical Analysis}

Agronomic characteristics and drought resistance index of the transgenic lines and WT were analyzed for their variation with respect to the effects of genotypes and treatments using the analysis of variance (ANOVA) by the SAS software version 8.0. The statistical significance of differences was analyzed based on the Fisher's LSD test.

\section{Results}

Overexpression of OsHox-6 Gene in The Transgenic Rice Plants

Real-time quantitative PCR (qRT-PCR) was performed to measure the expression of OsHox-6 in the leaves of the transgenic rice lines during water stress. The result showed that the expression of the OsHox-6 gene was increased in all the transgenic lines tested. The expression levels of OsHox-6 gene in these lines were 5 to 10 times higher than those of untransformed control plants (Figure 1) under a normal condition. When the leaves were imposed to dehydration, the expression of the OsHox-6 gene was even higher 10 to 20 times. The highest expression was found in the transgenic rice line S29-62-2.

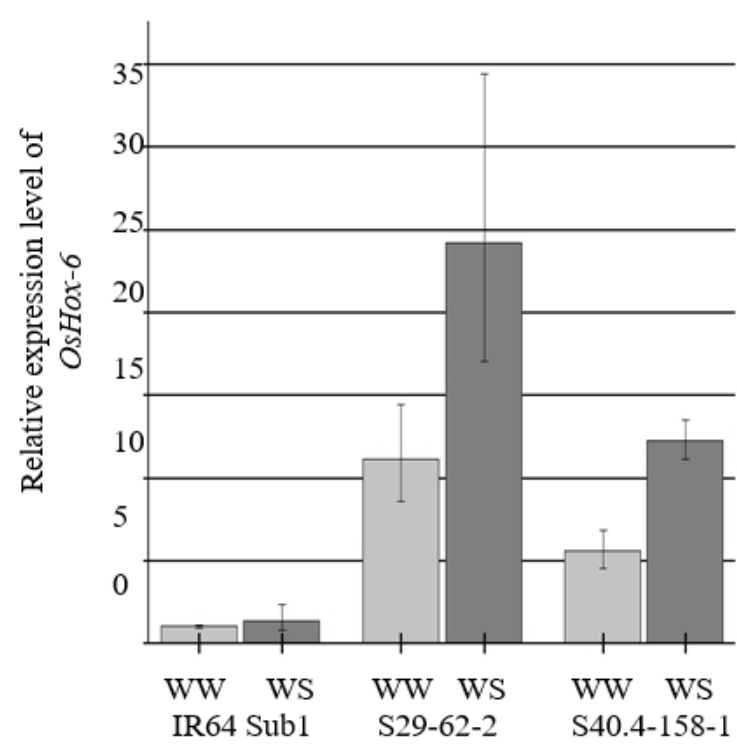

Figure 1. The relative expression of the OsHox-6 gene in two transgenic rice lines (S29-62-2 and S.40.4-158-1) revealed by a real-time quantitative PCR analysis. Total RNA was extracted from leaves before/wellwatered (WW) and after dehydrated for 1 hour at the room temperature/water stress (WS). Actin was used as a reference gene. Values represent the means of two replications.

\section{Phenotypes of OsHox-6 Overexpressed Transgenic Rice Lines}

Under a normal irrigation, we observed no significant differences between WT and OsHox-6 over-expressed transgenic lines in plant height. Interestingly, the tiller number of both transgenic lines were around 30-60\% more than the wild type. Likewise, the number of productive tillers of the transgenic lines were $25-60 \%$ more than that of WT. Meanwhile, the total grain number was significantly different between OsHox-6 overexpressed transgenic lines and its wild type. Unfortunately, the percentage of empty grains were also increased by 2-3 times in the transgenic lines. We also observed a 2-days delay of the flowering time in both transgenic lines. The phenotypes that are related to the important agronomic parameters are presented in the Table 2.

Overexpression of OsHox-6 Gene Did Not Significantly Improve the Water Loss Rate, Relative Water Content and Drought Resistance Index

There are a number of parameters that have been used to evaluate or select drought tolerant plants. Several drought tolerance mechanisms 
are associated with leaf water status such as transpirational water loss and relative water content. Water status of the plants that maintain cell turgor under water stress conditions are often used as the parameter to select drought tolerant or drought-avoidance plants (Hu \& Xiong, 2014). One simple and quick method to measure the rate of leaf water loss from plants is by using detached leaf method as described previously by Tang et al. (2012) and Yue et al. (2012).

Table 2. Agronomic characteristics of transgenic rice lines overexpressing OsHox-6 under standard irrigation conditions.

\begin{tabular}{cccc}
\hline & \multicolumn{3}{c}{ Lines } \\
\cline { 2 - 4 } Parameters & IR64 Sub1 & S29-62-2 & S40.4-158-1 \\
\hline PH $(\mathrm{cm})$ & $91,20 \pm 3.054 \mathrm{a}^{*}$ & $92,90 \pm 4.278 \mathrm{a}$ & $89,25 \pm 2.062 \mathrm{a}$ \\
TN & $12 \pm 2.1 \mathrm{a}$ & $16 \pm 4.0 \mathrm{ab}$ & $20 \pm 4.0 \mathrm{~b}$ \\
PTN & $12 \pm 2.2 \mathrm{a}$ & $15 \pm 4.1 \mathrm{ab}$ & $20 \pm 4.0 \mathrm{~b}$ \\
FT & 78 & 80 & 80 \\
TG & $1178 \pm 192.8 \mathrm{a}$ & $1536 \pm 375.9 \mathrm{~b}$ & $1715 \pm 336.0 \mathrm{~b}$ \\
FG & $1093 \pm 165.9 \mathrm{a}$ & $1284 \pm 268.1 \mathrm{a}$ & $1335 \pm 288.8 \mathrm{a}$ \\
$\%$ EG & $7,22 \pm 2.623 \mathrm{a}$ & $16,41 \pm 4.235 \mathrm{~b}$ & $22,17 \pm 6.892 \mathrm{~b}$ \\
SW1000 & $22,18 \pm 1.140 \mathrm{a}$ & $21,73 \pm 0.546 \mathrm{a}$ & $21,67 \pm 0.423 \mathrm{a}$ \\
\hline
\end{tabular}

Note: PH: plant height, TN: Tiller number, PTN: Productive tiller number, FT: Flowering time (days after transplanting), TG; total grain, FG: filled grain, EG: empty grain, SW1000: weight of 1000 seeds (gram).

* means sharing similar letter in a line do not differ significantly at $\alpha=0.05$

Using the detached leaf method, we monitored the rates of water loss from detached leaves of the transgenic rice lines overexpressing the OsHox6 gene over the time. In the beginning, the rates of water loss of the transgenic lines were slightly below those of the non-transgenic IR64 Sub1 at the seedling stage, but not significantly different. After $2 \mathrm{~h}$ exposure to dehydration, the rates of water loss of the transgenic lines were faster than those of the control wild type (Figure 2).

A similar trend was also observed on the relative water content (RWC). The measurement of the RWC was used to determine the hydration status of leaves relative to their maximal water holding capacity at full turgidity. Leaf water status of the transgenic rice lines and the IR64 Sub1 control under the well-watered condition were similar. When exposed to the water stress for 4 weeks, the RWC of the IR64 Sub1 control was decreased to less than $80 \%$ while the transgenic rice lines were able to maintain their RWC above $80 \%$ (Figure 3).

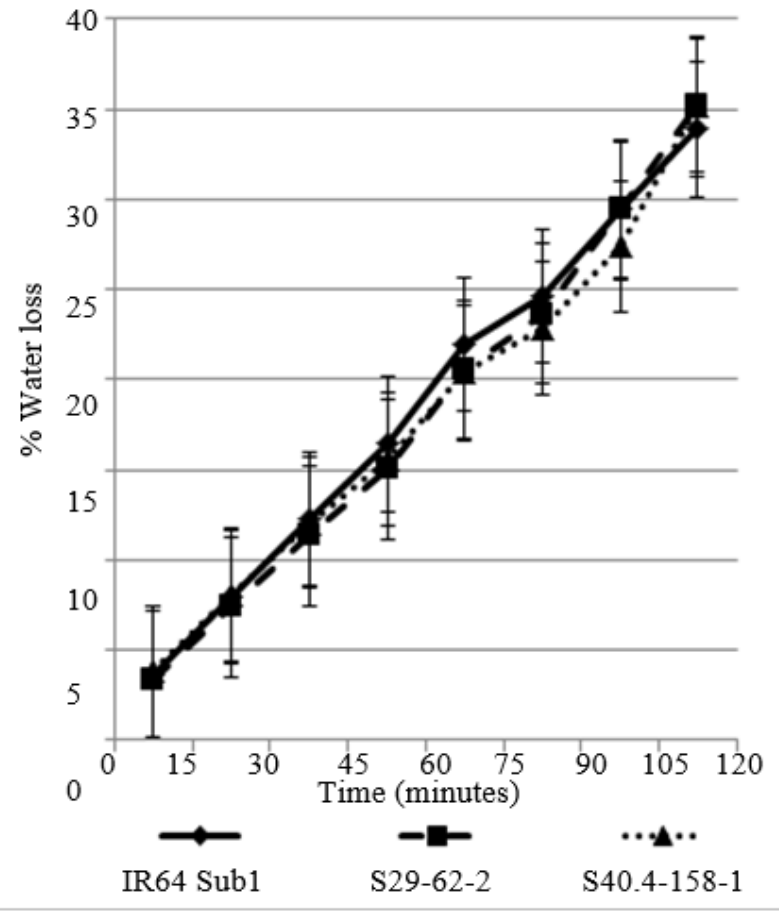

Figure 2. Comparison of water loss rates from detached shoots of the plants at the normal condition. Water loss represents the weight proportion of the water that loss by transpiration as compared to the initial weight. Values represent the means of 3 shoots from one plant. 
The reduction of RWC in the IR64 Sub1 control was, however, not significantly different with those of the transgenic rice lines, indicating that all the lines tested had relatively equal ability to maintain cell turgidity under the water stress condition.

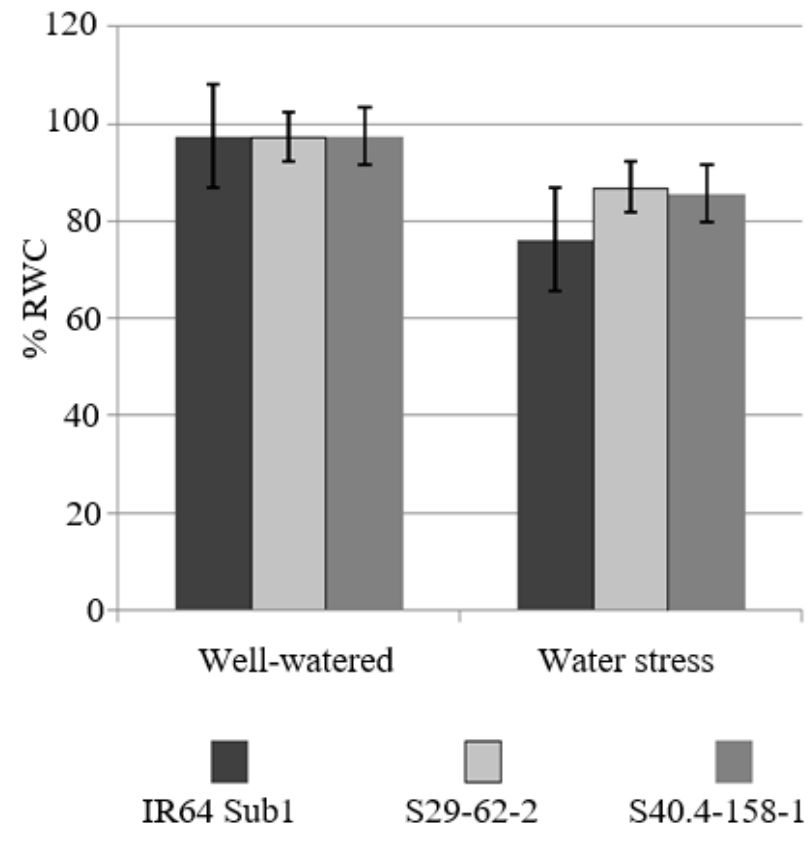

Figure 3. Leaf RWC of transgenic lines and IR64 Sub1 control under well- watered (WW) and water stress (WS) conditions.

Table 3. DRI of transgenic rice lines overexpressing OsHox6 and IR64 Sub1 control.

\begin{tabular}{lcl}
\hline \multicolumn{1}{c}{ Lines } & \multicolumn{2}{c}{ DRI } \\
& \multicolumn{1}{c}{ Biomass } & Total Grain \\
\hline IR 64 Sub 1 & $0.80 \pm 0.189 \mathrm{a}^{*}$ & $0.68 \pm 0.214 \mathrm{a}$ \\
S29-62-2 & $1.23 \pm 0.217 \mathrm{a}$ & $1.27 \pm 0.319 \mathrm{a}$ \\
S40.4-158-1 & $1.09 \pm 0.187 \mathrm{a}$ & $1.21 \pm 0.296 \mathrm{a}$ \\
\hline
\end{tabular}

Note: * means sharing similar letter in a column do not differ significantly at $\alpha=0.05$

Another criterion that commonly used to evaluate and select drought tolerant plants under drought condition is drought resistance index (DRI) (Farshadfar et al. 2013, Razzaq et al. 2013). This criterion has been considered as the best criterion for evaluation of resistant cultivars (Hu et al. 2007) and has been used as a standard in the identification of drought tolerant rice and wheat in China ( $\mathrm{Li}$ et al. 2006). Cultivars with greater DRI values are considered more resistant to drought.
The DRI values of the two homozygous lines tested in this experiment were presented in Table 3. The DRI was calculated based on the biomass and total grain (yield). The highest DRI was obtained for line S29-62-2 with DRI value of 1.23 and 1.27 for biomass and total grain, respectively. The DRI values were, however, not significantly different at $5 \%$ of probability.

\section{Effects of Drought on Growth Parameters}

Results from the observation on the agronomic characteristics of the transgenic lines overexpressing OsHox-6 and the wild type are presented in the Table 4. There was no significant difference in plant height between the transgenic lines with the WT before imposing to water stress. Plant height, however, was reduced significantly in transgenics as compared to the WT after the treatment (Figure 4, Table 4). We also observed that there were no significant differences in the tiller number and the productive tiller number between the transgenic lines and the WT IR64 Sub1 during the normal water condition. Meanwhile, the transgenic lines were able to recover faster than its wild type after the stress treatment by producing more tillers and productive tillers as indicated by the emergence of new panicles.

Observation on the yield parameters showed that under the well-watered conditions, transgenic lines produced total grain number less than the WT control. The total grain number in the transgenic lines tend to increase under the water-stress, whereas in the WT plants tend to decrease. However, under the water stress, there was no significant difference between the total grain number in the transgenic and non-transgenic lines Unfortunately, the percentage numbers of empty grains were also increased in transgenic plants overexpressing OsHox-6 grown under the normal and treatment conditions.

In the well-watered condition, the biomasses of rice transgenic lines were lower than that of the IR64 Sub1 control. However, after imposed to the water stress, the biomass of the non-transgenic IR64 Sub1 decreased to $25 \%$ while biomass production of the transgenic lines, S40.4-158-1 and S29-62-2, increased $2.5 \%$ and $15.2 \%$ respectively along with the increases of tiller numbers. 


\section{Discussion}

\section{Overexpression of OsHox6 Gene in The Transgenic Rice Plants Changed Plant Architechture}

Homeodomain leucine zipper (HD-Zip) transcription factors have been widely studied in the past few decades since they were found to be involved in various processes of plant growth and development in response to the environmental changes including abiotic (drought, salt, and osmotic stresses) and biotic stresses (virus) (Ariel et al. 2007, Ribone et al. 2015). HD-Zip transcription factors play a role as growth regulators in response to abiotic stresses including water stress.

Our results on this study also support that HDZip transcription factors, from which the OsHox-6 gene is one of the members of the subfamily 1, play a role in regulating plant growth as a response to water stress. Based on our data, the overexpression of OsHox-6 transcription factor did not significantly reduced plant height as previously reported on its homolog, including OsHox-22, OsHox-24, $A t H b-7$, and AtHb-12 (Hjellstrom et al. 2003, Olsson et al. 2004, Zhang et al. 2012, Bhattacharjee et al. 2016). However, the overexpression of OsHox-6 significantly increased tiller number, indicating that it might play an important role in tiller formation. Furthermore, we observed that several seeds germinated with 2-3 shoots (Rahmawati, 2012) where this phenomenon was not found for seeds originated from its WT control.

Similar results were previously reported by Hjellstrom et al. (2003) on Arabidopsis AtHB7 transcription factor, one of the homologs of OsHox-6 gene which had been well studied earlier. The Arabidopsis lines over-expressing AtHB-7 have more branches in the main inflorescence stem compared to the wild-type control, suggesting the decrease in apical dominance in the plants.

Tiller number and productive tiller number are two agronomic characteristics that are important in crop breeding to improve plant biomass and yield. Thus, finding the key genes in tiller formation would be very important to improve crops productivity (Zhang et al. 2009). Several genes involved in tiller formation have been found and characterized, including OsNPF-7.2 (Wang et al. 2018), OsMax-1 (Wang et al. 2015), and some other genes as described by Hussien et al. (2014). These genes may have interactions with or become the target of the OsHox-6 transcription factor. Thus, further research is still needed to discover the target genes of this transcription factor.

Table 4. Phenotypes of transgenic lines overexpressing OsHox-6 after exposed to water stress conditions.

\begin{tabular}{|c|c|c|c|c|}
\hline \multirow{2}{*}{\multicolumn{2}{|c|}{ Parameters }} & \multicolumn{3}{|c|}{ Lines } \\
\hline & & $\begin{array}{l}\text { IR } 64 \\
\text { Sub1 }\end{array}$ & S29-62-2 & $\begin{array}{c}\text { S40.4- } \\
158-1\end{array}$ \\
\hline \multirow{2}{*}{$\mathrm{PH}(\mathrm{cm})$} & WW & $\begin{array}{l}95.93 \pm \\
1.401 \mathrm{a}^{*}\end{array}$ & $\begin{array}{l}97.17 \pm \\
2.754 \mathrm{a}\end{array}$ & $\begin{array}{c}90.83 \pm \\
5.252 \mathrm{a}\end{array}$ \\
\hline & WS & $\begin{array}{c}89.83 \pm \\
2.485 \mathrm{a}\end{array}$ & $\begin{array}{l}87.43 \pm \\
2.542 \mathrm{ab}\end{array}$ & $\begin{array}{l}81.27 \pm \\
3.301 \mathrm{~b}\end{array}$ \\
\hline \multirow{2}{*}{$\mathrm{TN}$} & WW & $15 \pm 2.3 \mathrm{a}$ & $14 \pm 1.2 \mathrm{a}$ & $15 \pm 7.4 \mathrm{a}$ \\
\hline & WS & $14 \pm 1.7 \mathrm{a}$ & $21 \pm 2.1 b$ & $21 \pm 2.0 \mathrm{~b}$ \\
\hline \multirow{2}{*}{ PTN } & WW & $12 \pm 2.1 \mathrm{a}$ & $9 \pm 0.6 a$ & $11 \pm 3.5 \mathrm{a}$ \\
\hline & WS & $11 \pm 1.2 \mathrm{a}$ & $15 \pm 3.5 \mathrm{ab}$ & $16 \pm 1.0 \mathrm{~b}$ \\
\hline \multirow[b]{2}{*}{ TG } & WW & $\begin{array}{c}845 \pm \\
120.4 \mathrm{a}\end{array}$ & $\begin{array}{l}556 \pm \\
38.6 b\end{array}$ & $\begin{array}{c}564 \pm \\
125.8 b\end{array}$ \\
\hline & WS & $\begin{array}{l}511 \pm \\
86.3 \mathrm{a}\end{array}$ & $\begin{array}{c}628 \pm \\
183.1 \mathrm{a}\end{array}$ & $\begin{array}{c}606 \pm \\
143.5 \mathrm{a}\end{array}$ \\
\hline \multirow{2}{*}{ FG } & WW & $\begin{array}{l}568 \pm \\
177.7 \mathrm{a}\end{array}$ & $\begin{array}{c}434 \\
\pm 49.0 \mathrm{a}\end{array}$ & $\begin{array}{c}284 \pm \\
135.6 \mathrm{a}\end{array}$ \\
\hline & WS & $\begin{array}{l}321 \pm \\
29.9 \mathrm{a}\end{array}$ & $\begin{array}{c}231 \pm \\
147.3 \mathrm{ab}\end{array}$ & $\begin{array}{l}100 \pm \\
73.0 \mathrm{~b}\end{array}$ \\
\hline \multirow{2}{*}{$\% \mathrm{EG}$} & WW & $\begin{array}{c}32.78 \pm \\
20.044 a b\end{array}$ & $\begin{array}{c}21.94 \pm \\
3.556 \mathrm{a}\end{array}$ & $\begin{array}{l}49.64 \pm \\
14.039 b\end{array}$ \\
\hline & WS & $\begin{array}{l}37.18 \pm \\
16.668 \mathrm{a}\end{array}$ & $\begin{array}{l}63.22 \pm \\
28.495 b\end{array}$ & $\begin{array}{l}83.49 \pm \\
9.399 b\end{array}$ \\
\hline $\begin{array}{l}\text { Biomass } \\
\text { (g) }\end{array}$ & WW & $\begin{array}{c}103.52 \pm \\
14.149 \mathrm{a} \\
77.23 \pm \\
9.017 \mathrm{a}\end{array}$ & $\begin{array}{c}64.98 \pm \\
4.806 \mathrm{~b} \\
74.88 \pm \\
15.813 \mathrm{a}\end{array}$ & $\begin{array}{c}65.34 \pm \\
6.979 \mathrm{~b} \\
66.95 \pm \\
4.464 \mathrm{a}\end{array}$ \\
\hline
\end{tabular}

Note: PH: plant height, TN: Tiller number, PTN: Productive tiller number, TG; total grains, FG: filled grains, EG: empty grains, SW1000: weight of 1000 seeds. * means sharing similar letter in a line do not differ significantly at $\alpha=0.05$

Phylogenetically, OsHox-6 transcription factor is closely related with rice OsHox-22 and OsHox-24, and with Arabidopsis AtHb-7 and $A t H b-12$ as they are grouped in a same clade (Agalou et al. 2008, Zhang et al. 2014, Capella et al. 2016, Ribone et al. 2015). Presently, the OsHox-6 transcription factor has been known to share homology with the other genes in tomato (Solanum lycopersicum) SlHZ20, -32, -41 and -05 (Zhang et al. 2014); sunflower (Helianthus annuus) Hahb-11 and Hahb-4 (Ribone et al. 2015); wheat (Triticum 
aestivum) TaHDZ-8 dan -20 (Yue et al. 2018); and cassava (Manihot esculenta) MeHDZ-37, 38, -39, and -41 (Ding et al. 2017). These genes have been identified to be responsive to some abiotic stresses including drought. However, specific functions of most of these HD-Zip genes are still unknown.

Functional analyses of these homologous genes showed that they shared similar functions but also have specificities. Functional analysis studies showed that HDZip transcription factors act as negative growth regulators in response to drought. Rice OsHox-22 and its paralog, OsHox-24 for example, has a function as a negative growth regulator, specifically in root elongation in response to water stress via ABA-mediated signaling pathway. Overexpression of OsHox22 increased sensitivity to drought. Down regulation of OsHox-22, on the other hand, decreased sensitivity to ABA and increased drought tolerance. (Zhang et al. 2012, Bhattacharjee et al. 2016). Arabidopsis AtHB-7 and its paralog $A t H B-12$ also play a role as negative growth regulators in response to drought via ABA-mediated signaling pathway. Overexpression of both $A t H B-7$ and $A t H B-12$ reduced stem and leaf elongation, delayed senescence processes, and reduced stomatal conductance in mature plants (Olsson et al. 2004, Hjellstrom et al. 2003). Sunflower $H a h b-4$ plays a role as a negative regulator in cell elongation. Overexpression of Hahb-4 caused the plant had shorter stems and internodes, but became more tolerant to drought without yield penalty (Dezar et al. 2005). Overexpression Hahb-11 also induced root elongation, stem expansion and vascular bundle formation, increased plant tolerance to water stress. Since the overexpression of OsHox-6 did not significantly reduce plant height, this indicated that $O s H o x-6$ might not play a role in stem elongation. Previous studies on the fusion of a GUS promoter with rice OsHox-6 and Arabidopsis AtHB-7, respectively, showed that GUS activities were strongly induced by water stress (Hjellstrom et al. 2003, Rahmawati 2012). GUS activities of Arabidopsis AtHB7::GUS plants were most intense at the young part of inflorescences and the elongating parts of the stems (Hjellstrom et al. 2003). Whereas in rice OsHox-6::GUS plants, GUS activities were most intense in the divisional zone of stem nodes (Rahmawati, 2012).
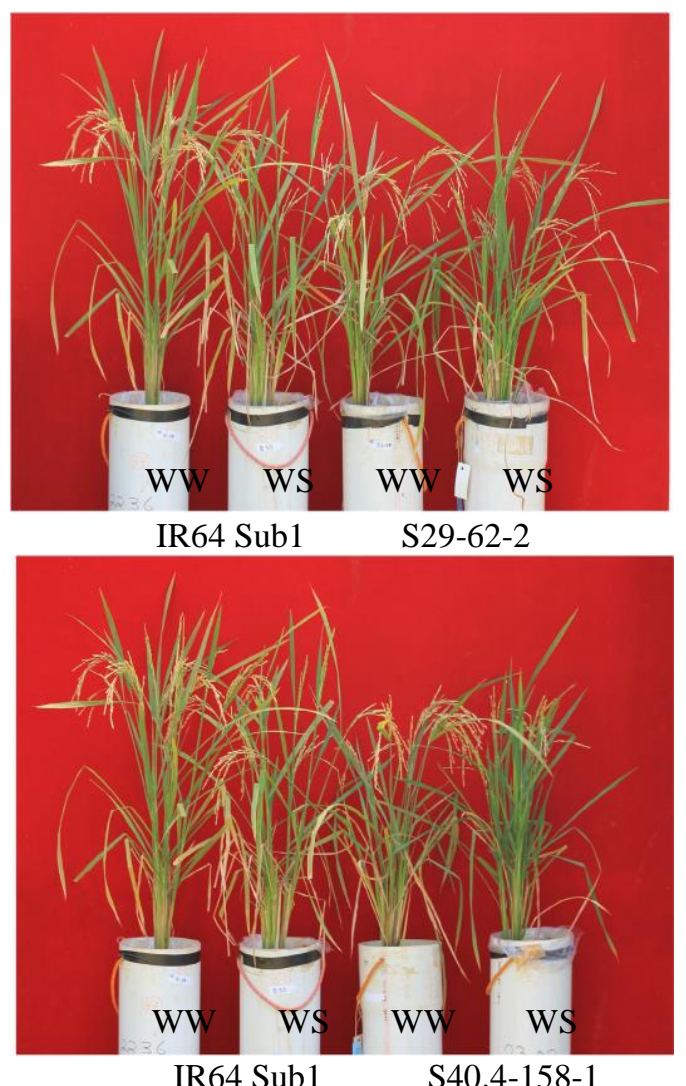

Figure 4. Comparison of transgenic rice lines overexpressing OsHox-6 and IR64 Sub1 control under well-watered (WW) and water stress (WS) conditions.

\section{Overexpression of OsHox-6 did not significantly increase tolerance to water stress.}

Based on our data, there was no significant difference between the transgenic overexpressing OsHox-6 and its control wild type in the water loss rates at the vegetative stage. No significant differences were also observed in the leaf water contents and drought resistance indeces (DRI) at the generative stage (Table 3). These results indicated that the overexpression of OsHox-6 might not significantly increase rice tolerance to the water stress. Furthermore, the overexpression of OsHox-6 delayed leaf senescence and caused yield penalty. These results were similar to what were observed in the other OsHox-6 homologs such as rice OsHox-22 and its ortholog OsHox-24, and also Arabidopsis $A t H B-7$ and its ortholog $A t H B-12$. The overexpression of OsHox-22, OsHox-24, $A t H B-7$ and $A t H B-12$ increased plant sensitivity to $\mathrm{ABA}$ and water stress, however, the decrease in their transcripts reduced 
sensitivity to $\mathrm{ABA}$ and increased tolerance to drought (Hjellstrom et al. 2003, Olsson et al. 2004, Zhang et al. 2012, Bhattacharjee et al. 2016). Thus, a down regulation or a knockdown of OsHox-6 expression is required to get a more comprehensive understanding on the role of the OsHox-6 gene in rice and its detailed mechanisms in response to drought and other abiotic stresses. Regulation of the OsHox-6 gene expression might be potential as a method to improve drought tolerance in rice and to shape rice architecture.

\section{Acknowledgements}

We acknowledge the support from Indonesian Institute of Science (LIPI) through National Priority Project (PN Pangan). Dr. Pieter B.F. Owerkerk from Leiden University for providing plasmid construct containing OsHox-6 gene. Dr. Inez H. Slamet Loedin from International Rice Research Institute (IRRI) for valuable suggestions and inputs as well as guidance in these studies.

\section{References}

Agalou, A., Purwantomo, S.E., Overnas, Johannesson, A., Zhu, X., Estiati, A., de Kam, R.J., Engstrom, P., Slamet-Loedin, I.H., Zhu, Z., Wang, M., Xiong, L., Meijer, A.H., \& Ouwerkerk, P.B.F. (2008). A genome-wide survey of HD-Zip genes in rice and analysis of drought-responsive family members. Plant Molecular Biology, 66:87-103.

Ariel, F.D., Manavella, P.A., Dezar, C.A., \& Chan, R.L. (2007). The true story of the HD-Zip family. Trends in Plant Science, 12:419-426.

Bhattacharjee, A., Khurana, J.P., \& Jain, M. (2016). Characterization of rice homeobox genes, OsHOX22 and OsHOX24, and overexpression of $\mathrm{OsHOX} 24$ in transgenic Arabidopsis suggest their role in abiotic stress response. Frontiers in Plant Sci., 7:627. doi: 10.3389/fpls.2016.00627

Capella, M., Ribone, P.A., Arce, A.L., \& Chan, R.L. (2016). Plant Transcription Factor First Edition. Chapter 7: Homeodomain-leucine zipper transcription factors: structural features of these proteins, unique to plants, 113-126. USA:Elsevier Inc.
Dai, M., Hu, Y., Ma, Q., Zhao, Y. \& Zhou, DX. (2008). Functional analysis of rice HOMEOBOX4 (Oshox4) gene reveals a negative function in gibberellin. Plant Molecular Biology, 66:289-301.

Deng, X.., Phillips, J., Meijer, A.H., Salamini, F. \& Bartels, D. (2002). Characterization of five novel dehydration-responsive homeodomain leucine zipper genes from the resurrection plant Craterostigma plantagineum. Plant Molecular Biology, 49:601-610.

Dezar, C.A., Gago, G.M., Gonzalez, D.H., \& Chan, R.L. (2005). HAHB-4, a sunflower homeo-box-leucine zipper gene, confers drought tolerance to Arabidopsis thaliana plants. Transgenic Research, 14:429-440.

Ding, Z., Fu, L., Yan, Y., Tie, W., Xia, Z., Wang, W., Peng, M., Hu, W., \& Zhang, J. (2017). Genome-wide characterization and expres-sion profiling of HD-Zip gene family related to abiotic stress in cassava. PLoS ONE,12(3):e0173043. doi:10.1371 /journal. pone.0173043

Farshadfar, E., Poursiahbidi, M.M., \& Safavi, S.M., (2013). Assesment of drought tolerance in land races of bread wheat based on resistance/tolerance indices. Iternational Journal of Advanced Biological and Biomedical Research, 1:143-158.

Fischer, R.A., \& Maurer, R. (1978). Drought resistance in spring wheat cultivars. I. Grain yield response. Australian Journal of Agricultural Research, 29: 897-907.

Frank W., Philips, J., Salamini, F., \& Bartels, D. (1998). Two dehidration inducible transcrip from the resurrection plant Craterostigma plantagineum encode interacting homeo-domain leucin zipper protein. The Plant Journal, 15:413-421.

Hjellstrom, M., Olsson, A.S.B., Engstrom, P., \& Soderman, E.M. (2003). Constitutive expression of the water deficit-inducible homeobox gene ATHB7 in transgenic Arabidopsis causes a suppression of stem elongation growth. Plant Cell \& Environment, 26:1127-1136.

Hu, S., Yang, H., Zou, G., Liu, H., Liu, G., Mei, H., Cai, R., Li M., \& Luo, L. (2007). Relationship between coleoptile length and drought resistance and their QTL mapping in rice. Rice Science, 14(1): 13-20.

$\mathrm{Hu}, \quad$ H., \& Xiong, L. (2014). Genetic engineering and breeding of drought- 
resistant crops. Annual Review of Plant Biology, 65:715-741.

Hussien, A., Tavakol, E., Horner, D.S., Munoz-Amatrianin, M., Muehlbauer, G.J., \& Rossini, L. (2014). Genetics of tillering in rice and barley. Plant Genome, 7:1-20. doi: 10.3835/ plantgenome2013.10.0032

Kholova, J., Hash, C.T., Kakkera, A., Kocova, M., \& Vadez, V. (2010). Constitutive water conserving mechanisms are correlated with the terminal drought tolerance of pearl millet [Pennisetum glaucum (L.) R. Br]. Journal of Experimental Botany, 61:369377.

Li, Y., Zhang, W., Xia, J., Pheng, H., Li, J., \& $\mathrm{Bu}$, J. (2006). Research and application of drought resistant identification index of major crop varieties. Acta Agriculture BorealiSinica, 21: 29-33.

Olsson, A.S.B., Engstrom, P., \& Soderman, E. (2004). The homeobox genes ATHB12 and $A T H B 7$ encode potential regulators of growth in response to water deficit in Arabidopsis. Plant Molecular Biology, 55: 663-677.

Prigge, M.J., Otsuga, D., Alonso, J.M., Ecker, J.R., Drews, G.N., \& Clarka, S.E. (2005). Class III homeodomain-leucine zipper gene family members have overlapping, antagonistic, and distinct roles in mitogenactivated protein kinase. Plant Cell, 17:6176.

Rahmawati, S. (2012). Genetic transformation of rice using Rhyzobium and Agrobacterium and functional analysis of OsHox-6 gene. (Doctoral Dissertation, Bogor: Bogor Agricultural University).

Razzaq, A., Ali, Q., Qayyum, A., Mahmood, I., Ahmad, M., \& Rasheed, M. (2013). Physiological responses and drought resistance index of nine wheat (Triticum aestivum L.) cultivars under different moisture conditions. Pakistan Journal of Botany, 45:151-155.

Rerie, W.G., Feldmann, K.A., \& Marks, M.D. (1994). The GLABRA2 gene encodes a homeo domain protein required for normal trichome development in Arabidopsis. Genes \& Development, 8:1388-1399.

Ribone, P.A., Capella, M., Arce, A.L., \& Chan, R.L. (2016). Plant Transcription Factors First Edition. Chapter 22: What do we know about homeodomain-leucine zipper I transcription factors? Functional and biotechnological considerations, 343-356. USA: Elsevier Inc.

Tang, N., Zhang, H., Li, X., Xiao, J., \& Xiong, L. (2012). Constitutive activation of transcript-ion factor OsbZIP46 improves drought tolerance in rice. Journal of Plant Physiology, 158:1755-1768.

Wang, X.M., Liang, Y.Y., Li, L., Gong, C.W., Wang, H.P., Huang, X.X., Li, S.C., Deng, Q.M., Zhu, J., Zheng, A.P., Li, P., Wang, S.Q. (2015). Identification and cloning of tillering-related genes $O s M A X 1$ in rice. Rice Science, 22(6): 255-263.

Wang, J., Lu, K.. Nie, H., Zeng, Q., Wu, B., Qian, J., \& Fang, Z. (2018). Rice nitrate transporter OsNPF7.2 positively regulates tiller number and grain yield. Rice, 11:12 https://doi.org/10.1186/s12284-018-0205-6

Xiao, B., Huang, Y., Tang, N., \& Xiong, L. (2007). Over-expression of a LEA gene in rice improves drought resistance under the field conditions. Theoretical and Applied Genetics, 115:35-46.

Yu, H., Chen, X., Hong, Y.Y., Wang, Y., Xu, P., Ke, S.D., Liu, H.Y., Zhu, J.K., Oliver, D.J. \& Xiang, C.B. (2008). Activated expression of an Arabidopsis HD-START protein confers drought tolerance with improved root system and reduced stomatal density. Plant Cell, 20:1134-1151.

Yue, Y., Zhang, M., Zhang, J., Tian, X., Duan, L., \& Li. Z. (2012). Overexpression of the AtLOS5 gene increased abscisic acid level and drought tolerance in transgenic cotton. Journal of Experimental Botany, 63(10):3741-3748. doi: 10.1093/jxb/ers069.

Yue, H., Shu, D., Wang, M., Xing, G., Zhan, H., Du, X., Song, W., \& Nie, X. (2018). Genome-Wide Identification and Expression Analysis of the HD-Zip Gene Family in Wheat (Triticum aestivum L.). Genes. 9(2). pii:E70. doi:10.3390/genes 9020070

Zhang, S.W., Li, C.H., Cao, J., Zhang, Y.C., Zhang, S.Q., Xia, Y.F., Sun, D.Y., \& Sun, Y. (2009). Altered architecture and enhanced drought tolerance in rice via down-regulation of Indole-3-Acetic Acid by TLD1/OsGH3.13 activation. Journal of Plant Physiology, 151:1889-1901.

Zhang, S., Haider, I., Kohlen, W., Jiang, L., Bouwmeester, H., Meijer, A.H., Schluepmann, H., Liu, C.M., Ouwerkerk, P.B.F. (2012). Function of the HD-Zip I gene Oshox22 in ABA-mediated drought 
and salt tolerances in rice. Plant Molecular Biology, 80(6):571-585. doi: 10.1007/ s11103-012-9967-1

Zhang, Z., Chen, X., Guan, X., Liu, Y., Chen, H., Wang, T., Mouekouba, L.D.O., Li, J., \& Wang, A. (2014). A genome-wide survey of homeodomain-leucine zipper genes and analysis of cold-responsive HD-Zip I members' expression in tomato, Bioscience Biotechnology and Biochemistry, 78(8): 1337-1349. doi: 10.1080/09168451.2014. 923292 